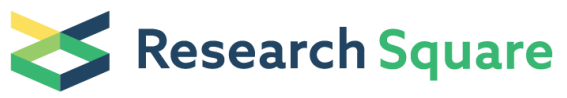 \\ Preprints are preliminary reports that have not undergone peer review. \\ They should not be considered conclusive, used to inform clinical practice, or referenced by the media as validated information.
}

\section{Characterization of Candidate Factors Associated With the Metastasis and Progression of High Grade Serous Ovarian Cancer}

\author{
Huiping Liu \\ Peking University Second School of Clinical Medicine: Peking University People's Hospital \\ Ling Zhou \\ Peking University Second School of Clinical Medicine: Peking University People's Hospital \\ Hongyan Cheng \\ Peking University Second School of Clinical Medicine: Peking University People's Hospital \\ Shang Wang \\ Peking University Second School of Clinical Medicine: Peking University People's Hospital \\ Wenqing Luan \\ Peking University Second School of Clinical Medicine: Peking University People's Hospital \\ E Cai \\ Peking University Second School of Clinical Medicine: Peking University People's Hospital \\ Ruiqiong Ma \\ Peking University Second School of Clinical Medicine: Peking University People's Hospital \\ Xue Ye \\ Peking University Second School of Clinical Medicine: Peking University People's Hospital \\ Heng Cui \\ Peking University Second School of Clinical Medicine: Peking University People's Hospital \\ Yi Li \\ Peking University Second School of Clinical Medicine: Peking University People's Hospital \\ xiaohong chang ( $\nabla$ changxiaohong@pkuph.edu.cn) \\ Peking University Second School of Clinical Medicine: Peking University People's Hospital
}

\section{Research Article}

Keywords: high grade serous ovarian cancer 1, metastasis 2, GEO 3, prognosis 4, recurrence 5, immune infiltration6

Posted Date: May 11th, 2021

DOl: https://doi.org/10.21203/rs.3.rs-497718/v1

License: (c) (1) This work is licensed under a Creative Commons Attribution 4.0 International License. Read Full License 


\section{Abstract}

Background. High grade serous ovarian cancer (HGSOC) is the highest cause of gynecological cancer-related mortality due to the extremely metastatic nature of this disease. The goal of this study is to explore and evaluate the profiles and characteristics of candidate factors associated with metastasis and progression of HGSOC.

Methods. Transcriptomic data of HGSOC patients' samples collected from the primary tumor and matched omental metastatic tumor were obtained from three independent studies in the NCBI GEO database. Genes significantly up-regulated and down-regulated were selected to evaluate the effects to prognosis and progression of ovarian cancer using data of ovarian cancer patients from The Cancer Genome Atlas (TCGA) database. Enrichment analysis for biological processes and pathways was performed using Gene Ontology (GO) and Kyoto Encyclopedia of Genes and Genomes(KEGG) analysis. Furthermore, the hub genes immune landscapes were estimated by Tumor Immune Estimation Resource (TIMER) database.

Results. 14 candidate genes included ADIPOQ, ALPK2, BARX1, CD37, CNR2, COL5A3, FABP4, FAP, GPR68, ITGBL1, MOXD1, PODNL1, SFRP2 and TRAF3IP3 were selected as up-regulated genes in metastatic tumors in every database while CADPS, GATA4, STAR and TSPAN8 were down-regulated. These 14 genes were significantly enriched for negative regulation of Wnt signaling pathway, fat cell differentiation, extracellular matrix organization. Finally, ALPK2, FAP, SFRP2 and GATA4, STAR, TSPAN8 were selected as hub genes that were found to be significantly associated with the survival and recurrence. All hub genes were correlated with several types of tumor microenvironmental cells infiltration significantly, especially for cancer associated fibroblasts and NK cells.

Conclusions. This study indicates that screening for differentially expressed genes and pathways in HGSOC primary tumor and matched metastasis tumor using integrated bioinformatics analyses. In sum, we identify six hub genes correlated with the progression of HGSOC in our study, which might provide effective targets to predict prognosis and provide novel insights into immune-based therapy strategies of HGSOC well.

\section{Background}

As the common cause of death related to gynecological cancer, ovarian cancer(OC) is the fifth cause of cancerous death among women all over the world, which leads to about $5 \%$ death of female cancer[1]. As the most aggressive and common histological subtype of ovarian cancer, the overall survival rate of High grade serous ovarian cancer(HGSOC) remains very dismal with $30 \%$ survival 5 years after the diagnosis since early detection is so difficult[2]. The main factor contributing to the high death to incidence rate is the advanced stage of the disease at the time of diagnosis[3]. Many patients are diagnosed after the tumor has already metastasized throughout the peritoneal cavity to other organizations, which typically occurs in the advanced HGSOC patients[4]. Commonly, HGSOC tumor cells undergo the processes of adhesion, migration, and invasion into the peritoneal cavity during metastasis and preferentially latch onto the omentum, which is the most common site of HGSOC metastasis [5, 6]. HGSOC progression and metastasis progress are facilitated by the networking interactions between cancer cells and various stromal components. The tumor microenvironment (TME) has been confirmed to play an important role in various cancer types, including OC. Worth mentioned, a vast array of factors such as immune response, metabolic pathways, intracellular signaling molecules shape the pathogenicity of TME, which in turn affects tumor progression, prognosis and even the efficacy of conventional treatment and immunotherapy[7]. Although, metastasis plays a crucial role in promoting OC progression and decreasing patient survival rates, but the underlying mechanisms of cancer cell spread have yet to be thoroughly explored and there is still a lack of comprehensive understanding of disease metastasis and progression. Hence, there will be of great value to understand the underlying molecular mechanisms of HGSOC metastasis.

In this study, aimed to identify the gene expression profiles associated with the progression of HGSOC, we employed a multistep bioinformatics strategy that used omics information and clinical data. We selected GSE30587, GSE137237, GSE98281, and GSE133296 from Gene Expression Omnibus (GEO) database according to the retrieval strategy and analyzed the genome-wide RNA-seq of HGSOC patients' samples collected from the primary tumor and matched omental metastatic tumor. Firstly, we successfully obtained 14 up-regulated genes and 4 down-regulated genes associated with HGSOC metastasis. Then, we validated the relationship between the genes and the progression of HGSOC by some clinical parameters and prognostic analysis. Finally, after a series of bioinformatics analyses, we selected ALPK2, FAP, SFRP2 and GATA4, STAR, TSPAN8 as the hub factors associated with the progression of HGSOC.

\section{Materials And Methods}

GEO datasets sources and processing

To identify the key genes involved in metastasis of HGSOC, we searched the key words (( "ovarian cancer" OR "ovarian carcinoma" [all fields] AND "metastasis" [all fields]) AND "Homo sapiens" [porgn]) in NCBI Gene Expression Omnibus firstly. Then, we screened the titles and abstracts of datasets, and the full information of the datasets of interest was further evaluated and finally selected according to the following inclusion criteria:(1) selected datasets should be mRNA transcriptome data; (2) only datasets containing more than 5 matched pairs of primary tumors and metastatic tumor samples from omentum were included; (3) the pathological type of the primary tumor is high grade serous carcinoma. Finally, we retrieved the RNA-seq data from 4 independent datasets in NCBI Gene Expression Omnibus (GSE98281, GSE137237, GSE133296 and GSE30587). The details for every dataset were shown in Table 1. 
Table 1

Details for GEO ovarian cancer data

\begin{tabular}{|llllll|}
\hline Reference & Cancer type & GEO & Platform & Primary tumor & omental metastasis \\
\hline Brodsky et al (2014) & HGSC & GSE30587 & GPL6244 & 9 & 9 \\
\hline Mitra et al(2019) & Serous adenocarcinoma & GSE137237 & GPL18573 & 11 & 11 \\
\hline Sallinen et al(2019) & HGSC & GSE98281 & GPL11154 & 10 & 10 \\
Hu et al(2020) & HGSC & GSE133296 & GPL20795 & 10 & 10 \\
\hline Abbreviation: HGSC: high-grade serous carcinoma & & & & \\
\hline
\end{tabular}

TCGA TARGET GTEx dataset analysis

UCSC Xena is an online exploration tool for multi-omic and clinical/phenotype data, which provides easy access to publicly available cancer transcriptome data including ovarian cancer to explore correlationships between genomic and/or phenotypic variables[8]. TCGA TARGET GTEX study(https://xenabrowser.net/) was used to compare differential genes expression among normal ovary tissues from The Genotype-Tissue Expression(GTEx), ovarian serous cystadenocarcinoma and recurrent tumors from The Cancer Genome Atlas(TCGA) to identify the impacts of candidate up and down-regulated DEGs on progression of ovarian cancer. We filtered the cohort and only kept the ovary samples cohort. The search term that was used to filter was: Ovary. Then we clicked to add columns for every candidate genes and choose the "sample type" option to compare the expression of the hub genes. In order to further explore the relationship of hub genes, log2 transformed RNA-sequencing data from 323 high grade serous ovarian cancer patients in TCGA dataset were also obtained through the UCSC Xena platform for correlation analysis (https://xenabrowser.net/datapages/).

Differential genes expression analysis

The differentially expressed genes (DEGs) were obtained by conducting differential expression analysis. The downloaded platform and series of matrix file(s) were converted using the $\mathrm{R}$ language software and annotation package. Gene differential expression analysis was performed using the limma package in the Bioconductor package (available online: http://www.bioconductor.org/). We divided the samples into two subgroups, namely primary tumor group(PT), metastasis tumor group(MT). By employing absolute $\mid \log _{2}$ fold change (FC) $\mid>1$ and $p$-value $<0.05$, the DEGs including significantly upregulated and downregulated genes between HGSOC primary tumor versus matched metastasis tissues in every GSE datasets were identified.

GO Term and KEGG Pathway Enrichment data analysis

With the screened DEGs, Metascape (http://www.metascape.org) was used to assess Gene Ontology(GO) functional annotation of DEGs and candidate genes in biological networks. GO chord was made by R language software. Kyoto Encyclopedia of Genes and Genomes (KEGG pathway) analyses of candidate genes were performed by KOBAS 3.0 online analysis database (http://kobas.cbi.pku.edu.cn/kobas3) to predict their underlying molecular functions. We set $p$ $<0.05$ as a significant criterion.

Kaplan-Meier plotter analysis

Kaplan-Meier plotter (https://kmplot.com) included ovarian cancer transcriptomic datasets in GEO and TCGA[9]. The overall survival analyses and progression-free survival analyses for up and down-regulated candidate genes were performed using Kaplan-Meier plotter with a hazard ratio (HR) with 95\% confidence intervals and log-rank $p$ value cutoff for each gene at 0.05 . And only patients diagnosed with serous histology were used for analysis.

TIMER2.0 database analysis

The Tumor Immune Estimation Resource (TIMER2.0) tool was a comprehensive online resource for analysis of immune cell infiltration among diverse cancers [10]. TIMER2.0 was used to analyze the correlation of hub genes with the infiltration level of several TME cells, including CD8 $+T$ cell, $C D 4+T$ cell, $B$ cell, myeloid dendritic cell, natural killer(NK) cell, macrophage, cancer associated fibroblast(CAF).

Statistical analysis

Student's t-tests were used to compare the expression level of PT group and MT group in GEO dataset. One way-ANOVA test was used for statistical comparison between multiple groups of boxplot. The log-rank test was used to calculate the HR and log-rank $p$ value to compare the survival curves. The correlation of hub genes expression and its relationship with TME cell infiltration levels was determined by Spearman's correlation. $\mathrm{p}$-values $<0.05$ were considered statistically significant.

\section{Results}

Screening of differentially expression genes associated with metastasis of HGSOC

To identify the key genes involved in metastasis and progression of HGSOC, we selected four GEO datasets, as GSE30587, GSE137237, GSE98281 and GSE133296 respectively according to our work flow as showed in Fig. 1 and Table 1. Every expression datasets were analyzed by the limma package and expression data were standardized, as the results were shown in Additional Figure S1. A total of 63 and 2 DEGs were significantly up and down-regulated in MT group compared with PT group in GSE30587 (Additional Table S1). Furthermore, 1884 DEGs were obtained from GSE137237 data, including 763 and 1121 DEGs were significantly up and down-regulated in MT group compared with PT group (Additional Table S2, Fig. 2a). Additionally, 465 DEGs were

Page $3 / 15$ 
screened from GSE98281 data. Among them, 193 and 272 DEGs were significantly up and down-regulated in MT group compared with PT group in (Additional Table S3, Fig. 2b). Besides, 1284 up-regulated genes and 1359 down-regulated genes were identified in GSE133296 according to the same principles of data processing as mentioned before (Additional Table S4, Fig. 2c). Considering that there were only 2 DEGs down-regulated in MT group in GSE30587, which would affect the overall data processing, the datasets we finally included were GSE137237, GSE90281, GSE133296.

In order to clarify the major characteristics of tumor metastasis process, we selected the up and down regulated DEGs of every dataset with $\|$ log ${ }_{2} \mathrm{FCl}>1.5$ to assess GO function enrichment. In GSE137237, the top highly enriched GO terms of up-regulated DEGs in MT group were extracellular matrix organization, vasculature development, connective tissue development and leukocyte migration (Fig. 2d,2f) specifically while cilium pathways were chiefly enriched for down-regulated DEGs (Fig. 2e-2g). In GSE98281, the top 5 enriched GO terms of up-regulated DEGs included lymphocyte activation, response to bacterium, regulation of cytokine production, negative regulation of immune system process, leukocyte differentiation (Fig. 2h-2j) while chemical synaptic transmission was enriched obviously for down-regulated DEGs (Fig. 2i-2k). In GSE133296, cornification and adaptive immune response were enriched distinctly (Fig. 2l-2n). Briefly, we found the pathways commonly enriched in up-regulated genes were focused on extracellular matrix and immune-related response programs.

Identification of candidate genes and function enrichment analysis

- This study aims to find those candidate genes associated with metastasis and progression of HGSOC. Therefore, as Fig. 3a, 3b shown, Venn diagrams showed 18 candidate genes which consisted of 14 up-regulated genes and 4 down-regulated genes in MT group that were commonly appeared in three comparison sets. Generally speaking, highly expressed genes in MT group might act as a carcinogenic effect. So, we concentrated on the characteristics of 14 up-regulated genes. The enriched GO terms of these genes were shown in Fig. 3c and Additional table S5, containing negative regulation of Wnt signaling pathway, fat cell differentiation, extracellular matrix organization, negative regulation of response to external stimulus. Furthermore, the enriched KEGG pathways were presented in Fig. 3d and Additional table S5 which included PPAR signaling pathway and multiple substances metabolism pathways like steroid and lipid. These enrichment pathways reminded us that the exchange of materials between tumor cells and the microenvironment might be frequent

Effects of individual candidate genes on OS and PFS

To explore the potential roles of the 18 candidate DEGs in overall survival(OS) and progression-free survival(PFS), Kaplan-Meier survival curves were used to establish the potential relationship between the prognostic roles and gene expression levels. Among the 18 DEGs, a total of 14 DEGs were found to be significantly related to OS in the log-rank test while 16 of 18 DEGs were linked with PFS. As shown in Fig. 4, among the 14 up-regulated candidate genes, we found that expression of 10 DEGs (ADIPOQ, ALPK2, BARX1, COL5A3, FABP4, FAP, GPR68, ITGBL1, MOXD1, SFRP2) was associated with OS of patients. Notably, except GPR68, the high expression of other 9 genes indicated a poor prognosis of ovarian patients. In this study, we mainly focused on the upregulated genes with high expression in MT group which might be correlated with unfavorable prognosis. So, the 9 genes (ADIPOQ, ALPK2, BARX1, COL5A3, FABP4, FAP, ITGBL1, MOXD1, SFRP2) were worthy of more attention.

For PFS, except CNR2 and TRAF3IP3, all other 12 up-regulated candidate genes were associated with short PFS of serous ovarian cancer patients as shown in Fig. 5a-5n. Additionally, the 4 low expressed genes (CADPS, GATA4, STAR, TSPAN8) in MT group were indicated good prognosis of patients not only with OS but also with PFS obviously.

Expression validation and recurrence evaluation of candidate genes in TCGA TARGET GTEx study

As we all known, HGSOC patients usually developed tumor recurrence which was the main reason for the poor prognosis. We compared the expression among normal-primary-recurrent tissues used TCGA TARGET GTEx dataset and found 15 of 18 candidate genes expression were significantly different. More importantly, our results showed that the expression of ALPK2, CD37, CNR2, FAP, GPR68, SFRP2 were reflected increased during tumor recurrence while GATA4, STAR and TSPAN8 were decreased.

Exploration of hub genes according to OS, PFS and recurrence

In our perspective, some key factors involved in metastatic process would have changed among normal-primary-recurrent progress. Meanwhile, it was well known that differentially gene expression patterns of metastasis are more likely to play key roles in the progression and prognosis of cancer. To dig up the hub genes precisely, we combined the effects of the candidate genes on OS, PFS and recurrence as shown in Table 2,3. We figured out the hub genes from 14 upregulated candidate genes in MT group with poor OS and PFS, and the expression of these genes in recurrent tumor was higher than that in primary tumors and normal tissues. The down-regulated genes were selected with good prognosis and lower expression level in the same way. In conclude, ALPK2, FAP, SFRP2 and GATA4, STAR, TSPAN8 were identified markedly. Moreover, Human Protein Atlas database was used to validate the protein expression of these hub genes as shown in Additional Figure S2a-e. ALPK2 and FAP was expressed membranous and cytosolic compartments in HGSOC tissues. In the same time, the association between the expression levels of the six genes from HGSOC patients in TCGA dataset was explored in Additional Figure S2f. The expression among ALPK2, FAP and SFRP2 was positively correlated, on the contrary, negatively correlated with the expression of GATA4, STAR and TSPAN8. 
Table 2

Summary for survival analysis and expression analysis of the 14 up-regulated genes

in metastasis tumors of OC patients

\begin{tabular}{|c|c|c|c|}
\hline Gene & OS(P®0.05) & PFS(P囚0.05) & expression(normal|"primary $\square$ recurrent) \\
\hline ADIPOQ & $\checkmark$ & $\checkmark$ & प \\
\hline ALPK2 & $\checkmark$ & $\checkmark$ & $\checkmark$ \\
\hline BARX1 & $\checkmark$ & $\checkmark$ & प \\
\hline CD37 & प & $\checkmark$ & $\checkmark$ \\
\hline CNR2 & प & 口 & $\checkmark$ \\
\hline COL5A3 & $\checkmark$ & $\checkmark$ & प \\
\hline FABP4 & $\checkmark$ & $\checkmark$ & प \\
\hline FAP & $\checkmark$ & $\checkmark$ & $\checkmark$ \\
\hline GPR68 & प & $\checkmark$ & $\checkmark$ \\
\hline ITGBL1 & $\checkmark$ & $\checkmark$ & प \\
\hline MOXD1 & $\checkmark$ & $\checkmark$ & प \\
\hline PODNL1 & प & $\checkmark$ & प \\
\hline SFRP2 & $\checkmark$ & $\checkmark$ & $\checkmark$ \\
\hline TRAF3IP3 & प & प & $\square$ \\
\hline
\end{tabular}

Table 3

Summary for survival analysis and expression analysis of the 4 down-regulated genes in metastasis tumors of OC patients.

\begin{tabular}{|c|c|c|c|}
\hline Gene & OS(Pष0.05) & PFS(Pख0.05) & expression(normal[primary[recurrent) \\
\hline CADPS & $\checkmark$ & $\checkmark$ & प \\
\hline GATA4 & $\checkmark$ & $\checkmark$ & $\checkmark$ \\
\hline STAR & $\checkmark$ & $\checkmark$ & $\checkmark$ \\
\hline TSPAN8 & $\checkmark$ & $\checkmark$ & $\checkmark$ \\
\hline
\end{tabular}

Relationship between the expression of hub genes and tumor microenvironmental cells infiltration level

As mentioned before, the interplay among metastatic tumor cells and different cell types within the TME was very close in the process of tumor metastasis. So, we examined the correlation between the expression of hub genes and the differential abundance of tumor microenvironmental cells infiltration, especially for six types of immune cells and cancer associated fibroblast infiltration by TIMER2.0 database. As Additional Figure S3 and Table 4 presented, every of the hub genes was correlated with tumor purity in OC tissues. Notably, we observed that three up-regulated hub genes (ALPK2, FAP, SFRP2) presented positive associations significantly with infiltrating levels of CD $8+T$ cells, B cells, macrophages, NK cells and CAFs, out of which these genes most strongly correlated with CAFs [correlation coefficient (COR), 0.736 to $0.83 ; p<0.001$ ], NK cells (COR, 0.238 to $0.325 ; p<0.001$ ), and macrophages (COR 0.238 to $0.244 ; p<0.001$ ). On the contrary, down-regulated hub genes (GATA4, STAR, TSPAN8) were negatively correlated with the infiltration of NK cells $(-0.29$ to $-0.33 ; p<0.001)$ and CAFs (COR, -0.237 to $-0.324 ; p<0.001)$ (Fig. 7). That indicated that these hub genes were specifically related to cancer-associated NKs and CAFs in the OC microenvironment. 
Table 4

The relationship between the expression of 6 hub genes and tumor microenvironmental cells infiltration level

\begin{tabular}{|c|c|c|c|c|c|c|c|c|c|c|c|c|c|c|c|}
\hline \multirow{2}{*}{$\begin{array}{l}\text { Hub } \\
\text { genes }\end{array}$} & \multicolumn{2}{|l|}{ Purity } & \multicolumn{2}{|c|}{ CD8 $+\mathrm{T}$ cell } & \multicolumn{2}{|c|}{ CD4 + T cell } & \multicolumn{2}{|l|}{ B cell } & \multicolumn{2}{|l|}{ DC cell } & \multicolumn{2}{|l|}{ NK cell } & \multicolumn{2}{|c|}{ Marcrophage } & \multirow{2}{*}{$\frac{\mathrm{CAF}}{\mathrm{COI}}$} \\
\hline & COR & $\mathbf{P}$ & COR & $\mathbf{P}$ & COR & $P$ & COR & $P$ & COR & $\mathbf{P}$ & COR & $\mathbf{P}$ & COR & $P$ & \\
\hline ALPK2 & -0.519 & $\begin{array}{l}1.24 \mathrm{e} \\
-18\end{array}$ & 0.203 & $\begin{array}{l}1.26 \mathrm{e} \\
-03\end{array}$ & -0.055 & 0.391 & -0.187 & $\begin{array}{l}3.12 e \\
-03\end{array}$ & -0.005 & 0.936 & 0.302 & $\begin{array}{l}1.23 e \\
-06\end{array}$ & 0.243 & $\begin{array}{l}1.08 \mathrm{e} \\
-04\end{array}$ & 0.81 \\
\hline FAP & -0.595 & $\begin{array}{l}2.77 e \\
-25\end{array}$ & 0.196 & $\begin{array}{l}1.85 e \\
-03\end{array}$ & -0.045 & 0.479 & -0.222 & $\begin{array}{l}4.05 e \\
-04\end{array}$ & 0.035 & 0.583 & 0.325 & $\begin{array}{l}1.62 \mathrm{e} \\
-07\end{array}$ & 0.238 & $\begin{array}{l}1.50 \mathrm{e} \\
-04\end{array}$ & $0.8:$ \\
\hline SFRP2 & -0.5 & $\begin{array}{l}3.04 \mathrm{e} \\
-17\end{array}$ & 0.19 & $\begin{array}{l}2.64 \mathrm{e} \\
-03\end{array}$ & -0.094 & 0.137 & -0.245 & $\begin{array}{l}9.50 \mathrm{e} \\
-05\end{array}$ & -0.064 & 0.313 & 0.238 & $\begin{array}{l}1.55 \mathrm{e} \\
-04\end{array}$ & 0.244 & $\begin{array}{l}1.02 \mathrm{e} \\
-04\end{array}$ & $0.7:$ \\
\hline GATA4 & 0.138 & 0.0289 & -0.125 & 0.0494 & 0.044 & 0.489 & 0.025 & 0.691 & -0.14 & 0.027 & -0.29 & $\begin{array}{l}3.32 \mathrm{e} \\
-06\end{array}$ & -0.208 & $\begin{array}{l}9.45 e \\
-04\end{array}$ & $-0 .{ }^{\prime}$ \\
\hline STAR & 0.281 & $\begin{array}{l}6.61 e \\
-06\end{array}$ & -0.179 & $\begin{array}{l}4.65 e \\
-03\end{array}$ & 0.091 & 0.151 & 0.04 & 0.526 & -0.138 & 0.0298 & -0.297 & $\begin{array}{l}1.88 \mathrm{e} \\
-06\end{array}$ & -0.2 & $\begin{array}{l}1.52 \mathrm{e} \\
-03\end{array}$ & $-0 . x^{\prime}$ \\
\hline TSPAN8 & 0.224 & $\begin{array}{l}3.57 e \\
-04\end{array}$ & -0.115 & 0.0698 & 0.011 & 0.862 & 0.001 & 0.993 & -0.105 & 0.0984 & -0.33 & $\begin{array}{l}1.02 e \\
-07\end{array}$ & 0.007 & 0.917 & $-0 .{ }^{\prime}$ \\
\hline
\end{tabular}

\section{Discussion}

HGSOC is the most common histologic subtype of OC, with a poor 5 -year survival rate of $35-40 \%$ and accounting for $70-80 \%$ of $0 \mathrm{C}$ deaths[1]. Although surgery and other treatment methods have been improved, the treatment effect and prognosis of HGSOC patients are very poor due to the late diagnosis [11]. Because about $70 \%$ of $\mathrm{OC}$ cases are diagnosed at a late stage, when cancer cells are actively metastasizing, understanding ovarian cancer pathogenesis and the mechanism of its metastasis is crucial for the management of this deadly, highly metastatic disease[5, 12]. Although multiple level studies have been carried out, they are not yet mature. Thus, there is still a need to screen novel metastasis associated factors to better understand the mechanism.

We conducted this study to identify more useful metastatic factors in HGSOC used bioinformatical methods on the basis of three profile datasets (GSE137237, GSE98281 and GSE133296). We compared the differential gene expression of metastatic tumors and primary tumors in every dataset. Our results showed that the main prominent features of up-regulated DEG were extracellular matrix, regulation of immune response and angiogenesis, etc. Many researchers have demonstrated that ovarian cancer metastasis from the primary site to the omental metastatic tumor site are facilitated by the interaction between cancer cells and various microenvironmental components [13,14]. After ovarian cancer cell implantation, the resultant inflammation and injury stimulate the peritoneal cells and their associated immune and stromal cells to release cytokines which further leads to tumor progression [15].

In our study, we took the intersection of the DEGs of the above three datasets, and these datasets shared 18 common DEGs in MT group contained 14 upregulated genes and 4 down-regulated genes. Following this, the GO and KEGG analysis was constructed focused on up-regulated genes to describe metastasis characteristics intensively. On one hand, we found that the $\mathrm{GO}$ terms of 14 candidate genes were particularly enriched in negative regulation of Wnt signaling pathway and fat cell differentiation. As we all known, the Wnt/ $\beta$-catenin signaling pathways play a crucial role in tumorigenesis, metastasis, and therapy resistance of OC subtypes, especially for HGSOC [16-18]. Alexandra McMellen et al demonstrated that the expression of typical Wnt molecules is dependent on anatomic/metastatic site, highlighting the importance of the TME and indicating that Wnt signaling activity in HGSOC varies depending on this TME [19]. Recent studies highlighted the understanding of the relevance of altered lipid metabolic pathways contributing to the poor prognosis of HGSOC. Cancer cells utilize various strategies to boost lipid uptake in order to fulfil their high energetic needs for cell growth and altered oncogenic signaling [20,21]. Meanwhile, cancer-associated adipocytes promote the growth of cancer cells mediated by adipokines including interleukin-8 (IL-8) and provide energy for rapid tumor growth $[22,23]$. On the other hand, KEGG pathway enrichment of above genes were PPAR signaling pathway and multiple substances metabolism especially for lipid. In current, PPAR exerts multiple regulatory functions of the metabolism of lipid, glucose, and amino acid, as well as inflammation, cell proliferation, and apoptosis in cancer [24]. Mukherjee, A, et al. reported that FABP4, which was mediated by PPAR, was upregulated in omental metastases and suggested as a pro-tumorigenic role in ovarian cancer [25]. This is consistent with our results and suggests us to focus on new strategies targeting key enzymes involved in lipid uptake or utilization in cancer cells.

To better identify the key factors of metastasis, the intersected genes among common DEGs, prognosis and recurrence-related genes sifted out particularly. Finally, six hub genes were selected, as ALPK2, FAP, SFRP2 were upregulated in HGSOC metastatic tissues respectively while GATA4, STAR, TSPAN8 were deregulated. It was known that ALPK2 played a vital role in cancer by regulating cell cycle and DNA repair and it was pointed out that knockdown of ALPK2 could inhibit proliferation, migration and promote apoptosis, arrested cell cycle of OC cells[26, 27]. FAP is overexpressed by fibroblasts present in the microenvironment of many tumors. Many studies have found that high expression of FAP in EOC is associated with poorer clinical outcomes [28, 29]. FAP may have novel cell-autonomous effects suggesting that targeting FAP could have pleiotropic anti-tumor effects. Notably, SFRP2, served as a potent regulator of Wnt signaling, can stimulate angiogenesis of cancer, accelerate tumor metastasis formation[30]. Therefore, some researchers highlight that SFRP2 has a potential role as anti-angiogenic therapeutic target in cancer. Significantly, Mariani Andrea reported that FAP and SFRP2 were both overexpressed in bowel metastases compared with primary tumors among patients with OC [31]. It reminded us that FAP and SFRP2 should be studied as potential therapeutic targets to decrease implantation or growth of OC cells. Interestingly, some researches confirmed that loss of expression and epigenetic silencing of the GATA4 gene could play an important role in ovarian carcinogenesis [32,33]. Abd-Elaziz $M$ et al reported that STAR expression appeared to confer a better prognosis of the patients of ovarian carcinoma [34]. And TSPAN8 could be therapeutic target in epithelial ovarian cancer invasion and metastasis[35]. But more exploration of mechanism and function experiments of hub genes in $\mathrm{OC}$ were still ambiguous. In a word, these researches highlighted the value of these hub genes for the progress of OC. 
Besides malignant transformed cells, tumors are composed of other cells including fibroblasts, muscle cells, immune cells and so on, altogether generating the TME. The TME is a remarkable complex ecosystem and components participate in numerous cancer progresses, such as tumorigenesis, metastasis [36, 37], response to chemotherapy and immune checkpoint blocking therapy[38], and prediction of clinical outcomes[39, 40]. Our results showed that six hub genes presented associations significantly with infiltrating levels of NK cells and CAFs. Generally speaking, CAFs are the most abundant cell type in the TME and greatly influenced immune cell activity and function within the TME [41, 42]. And the involvement of NK cells in OC was multifaceted as they could exert pro-tumor or anti-tumor effects. The functional capability of NK cells may be strongly impaired by the tumor microenvironment [43]. Activated CAFs are known to support tumor growth and secrete chemokines and cytokines like TGF $\beta$, which can effectuate immunosuppression and inhibit cytokine secretion from NK cells, thus decreasing its cytotoxicity[44]. Although the molecular mechanism of the NK cell-CAF interactions is still not clear and lack of previous tests in combination, these results demonstrate us that targeting tumor-promoting CAFs and exploiting NK cells, separately, are beneficial as therapeutic strategies. As of so far, although there were 28 clinical trials registered in ClinicalTrials.gov about NK cells therapy and ovarian cancer, the data of ovarian cancer was still limited yet. And it is worth exploring how to exploit NK cell cytotoxicity in tumors and how to target CAFs to enhance efficacy of cancer therapies and cytotoxic immune cells.

However, several limitations in our study should be acknowledged. Our study provides the evidence that six novel genes are significantly related to the progression of HGSOC patients and possibly become therapeutic targets for precision medicine in the future, which was analyzed through data mining merely. Further functional experiment for revealing their roles in cancers is valuable and crucial.

\section{Conclusions}

In conclusion, HGSOC is undoubtedly a challenge for patients, medical practitioners, and researchers because of its lethality. In this study, we identified six hub genes (ALPK2, FAP, SFRP2, GATA4, STAR, TSPAN8) associated with metastasis and progression of HGSOC and also explored the relationship between the genes and TME cells infiltration. This study will help us understand the underlying mechanisms involved in HGSOC and develop a clinical assay for guiding therapeutic selection and individualized treatment.

\section{Abbreviations}

HGSOC: High grade serous ovarian cancer; OC: ovarian cancer; TCGA: The Cancer Genome Atlas; GEO: Gene Expression Omnibus; TME: tumor microenvironment; GO: Gene Ontology; KEGG: Kyoto Encyclopedia of Genes and Genomes; GTEx: Genotype-Tissue Expression; DEGs: differentially expressed genes; HR: hazard ratio; NK: natural killer; CAF: cancer associated fibroblast; OS: overall survival; PFS: progression-free survival.

\section{Declarations}

\section{Ethics approval and consent to participate}

Not applicable.

\section{Consent for publication}

Not applicable.

\section{Availability of data and material}

All data generated or analysed during this study are included in this published article and its supplementary information files.

\section{Competing interests}

The authors declare that the research was conducted in the absence of any commercial or financial relationships that could be construed as a potential conflict of interest.

\section{Funding}

This work was financially supported by National Key Research and Development Program of China (nos. 2016YFA0201404).

\section{Author Contributions}

Huiping Liu: designed the study, analyzed the data and wrote the manuscript. Ling Zhou: completed the quality control and analyzed the data. Huiping Liu and Ling Zhou had equal contributions to this manuscript. Hongyan Cheng, Shang Wang, Wenqing Luan, E Cai, Ruiqiong Ma, Xue Ye and Heng Cui contributed to the writing of the manuscript. Xiaohong Chang and Yi Li performed the research and supervised the manuscript. All authors contributed to the article and approved the submitted version.

\section{Acknowledgments}

Thanks to the GEO, TCGA, UCSC Xena and TIMER databases for open data

\section{References}


1. Siegel, R.L., K.D. Miller, H.E. Fuchs, and A. Jemal, Cancer Statistics, 2021. CA Cancer J Clin, 2021. 71(1): p. 7-33.

2. Lheureux, S., M. Braunstein, and A.M. Oza, Epithelial ovarian cancer: Evolution of management in the era of precision medicine. CA Cancer J Clin, 2019. 69(4): p. 280-304.

3. Nik, N.N., R. Vang, M. Shih le, and R.J. Kurman, Origin and pathogenesis of pelvic (ovarian, tubal, and primary peritoneal) serous carcinoma. Annu Rev Pathol, 2014. 9: p. 27-45.

4. Ferraresi, A., C. Girone, A. Esposito, C. Vidoni, L. Vallino, E. Secomandi, et al., How Autophagy Shapes the Tumor Microenvironment in Ovarian Cancer. Front Oncol, 2020. 10: p. 599915.

5. Yeung, T.L., C.S. Leung, K.P. Yip, C.L. Au Yeung, S.T. Wong, and S.C. Mok, Cellular and molecular processes in ovarian cancer metastasis. A Review in the Theme: Cell and Molecular Processes in Cancer Metastasis. Am J Physiol Cell Physiol, 2015. 309(7): p. C444-56.

6. Bilbao, M., J.K. Aikins, and O. Ostrovsky, Is routine omentectomy of grossly normal omentum helpful in surgery for ovarian cancer? A look at the tumor microenvironment and its clinical implications. Gynecol Oncol, 2021. 161(1): p. 78-82.

7. Lengyel, E., Ovarian cancer development and metastasis. Am J Pathol, 2010. 177(3): p. 1053-64.

8. Goldman, M.J., B. Craft, M. Hastie, K. Repečka, F. McDade, A. Kamath, et al., Visualizing and interpreting cancer genomics data via the Xena platform. Nat Biotechnol, 2020. 38(6): p. 675-678.

9. Gyorffy, B., A. Lánczky, and Z. Szállási, Implementing an online tool for genome-wide validation of survival-associated biomarkers in ovarian-cancer using microarray data from 1287 patients. Endocr Relat Cancer, 2012. 19(2): p. 197-208.

10. Li, T., J. Fu, Z. Zeng, D. Cohen, J. Li, Q. Chen, et al., TIMER2.0 for analysis of tumor-infiltrating immune cells. Nucleic Acids Res, 2020. 48(W1): p. W509w514.

11. Bowtell, D.D., S. Böhm, A.A. Ahmed, P.J. Aspuria, R.C. Bast, Jr., V. Beral, et al., Rethinking ovarian cancer Il: reducing mortality from high-grade serous ovarian cancer. Nat Rev Cancer, 2015. 15(11): p. 668-79.

12. Kostova, I., R. Mandal, S. Becker, and K. Strebhardt, The role of caspase-8 in the tumor microenvironment of ovarian cancer. Cancer Metastasis Rev, 2021. 40(1): p. 303-318.

13. Motohara, T., K. Masuda, M. Morotti, Y. Zheng, S. El-Sahhar, K.Y. Chong, et al., An evolving story of the metastatic voyage of ovarian cancer cells: cellular and molecular orchestration of the adipose-rich metastatic microenvironment. Oncogene, 2019. 38(16): p. 2885-2898.

14. Worzfeld, T., E. Pogge von Strandmann, M. Huber, T. Adhikary, U. Wagner, S. Reinartz, et al., The Unique Molecular and Cellular Microenvironment of Ovarian Cancer. Front Oncol, 2017. 7: p. 24.

15. De Nola, R., A. Menga, A. Castegna, V. Loizzi, G. Ranieri, E. Cicinelli, et al., The Crowded Crosstalk between Cancer Cells and Stromal Microenvironment in Gynecological Malignancies: Biological Pathways and Therapeutic Implication. Int J Mol Sci, 2019. 20(10).

16. Gatcliffe, T.A., B.J. Monk, K. Planutis, and R.F. Holcombe, Wht signaling in ovarian tumorigenesis. Int J Gynecol Cancer, 2008. 18(5): p. 954-62.

17. Teeuwssen, M. and R. Fodde, Wnt Signaling in Ovarian Cancer Stemness, EMT, and Therapy Resistance. J Clin Med, 2019. 8(10).

18. Nguyen, V.H.L., R. Hough, S. Bernaudo, and C. Peng, Wnt/ $\beta$-catenin signalling in ovarian cancer: Insights into its hyperactivation and function in tumorigenesis. J Ovarian Res, 2019. 12(1): p. 122.

19. McMellen, A., E.R. Woodruff, B.R. Corr, B.G. Bitler, and M.R. Moroney, Wnt Signaling in Gynecologic Malignancies. Int J Mol Sci, 2020. 21(12).

20. Yu, C., X. Niu, Y. Du, Y. Chen, X. Liu, L. Xu, et al., IL-17A promotes fatty acid uptake through the IL-17A/IL-17RA/p-STAT3/FABP4 axis to fuel ovarian cancer growth in an adipocyte-rich microenvironment. Cancer Immunol Immunother, 2020. 69(1): p. 115-126.

21. Zhao, J., X. Zhang, T. Gao, S. Wang, Y. Hou, P. Yuan, et al., SIK2 enhances synthesis of fatty acid and cholesterol in ovarian cancer cells and tumor growth through PI3K/Akt signaling pathway. Cell Death Dis, 2020. 11(1): p. 25.

22. Nieman, K.M., H.A. Kenny, C.V. Penicka, A. Ladanyi, R. Buell-Gutbrod, M.R. Zillhardt, et al., Adipocytes promote ovarian cancer metastasis and provide energy for rapid tumor growth. Nat Med, 2011. 17(11): p. 1498-503.

23. Zhao, G., H. Cardenas, and D. Matei, Ovarian Cancer-Why Lipids Matter. Cancers (Basel), 2019. 11(12).

24. Tan, Y., M. Wang, K. Yang, T. Chi, Z. Liao, and P. Wei, PPAR-a Modulators as Current and Potential Cancer Treatments. Front Oncol, $2021.11:$ p. 599995.

25. Mukherjee, A., C.Y. Chiang, H.A. Daifotis, K.M. Nieman, J.F. Fahrmann, R.R. Lastra, et al., Adipocyte-Induced FABP4 Expression in Ovarian Cancer Cells Promotes Metastasis and Mediates Carboplatin Resistance. Cancer Res, 2020. 80(8): p. 1748-1761.

26. Yoshida, Y., T. Tsunoda, K. Doi, T. Fujimoto, Y. Tanaka, T. Ota, et al., ALPK2 is crucial for luminal apoptosis and DNA repair-related gene expression in a three-dimensional colonic-crypt model. Anticancer Res, 2012. 32(6): p. 2301-8.

27. Zhu, X., S. Yan, S. Xiao, and M. Xue, Knockdown of ALPK2 inhibits the development and progression of Ovarian Cancer. Cancer Cell Int, $2020.20:$ p. 267.

28. Li, M., X. Cheng, R. Rong, Y. Gao, X. Tang, and Y. Chen, High expression of fibroblast activation protein (FAP) predicts poor outcome in high-grade serous ovarian cancer. BMC Cancer, 2020. 20(1): p. 1032.

29. Mhawech-Fauceglia, P., L. Yan, M. Sharifian, X. Ren, S. Liu, G. Kim, et al., Stromal Expression of Fibroblast Activation Protein Alpha (FAP) Predicts Platinum Resistance and Shorter Recurrence in patients with Epithelial Ovarian Cancer. Cancer Microenviron, 2015. 8(1): p. $23-31$.

30. van Loon, K., E.J.M. Huijbers, and A.W. Griffioen, Secreted frizzled-related protein 2: a key player in noncanonical Wnt signaling and tumor angiogenesis. Cancer Metastasis Rev, 2021. 40(1): p. 191-203.

31. Mariani, A., C. Wang, A.L. Oberg, S.M. Riska, M. Torres, J. Kumka, et al., Genes associated with bowel metastases in ovarian cancer. Gynecol Oncol, 2019. 154(3): p. 495-504. 
32. Bubancova, I., H. Kovarikova, J. Laco, E. Ruszova, O. Dvorak, V. Palicka, et al., Next-Generation Sequencing Approach in Methylation Analysis of HNF1B and GATA4 Genes: Searching for Biomarkers in Ovarian Cancer. Int J Mol Sci, 2017. 18(2).

33. Baranova, I., H. Kovarikova, J. Laco, I. Sedlakova, F. Vrbacky, D. Kovarik, et al., Identification of a four-gene methylation biomarker panel in high-grade serous ovarian carcinoma. Clin Chem Lab Med, 2020. 58(8): p. 1332-1340.

34. Abd-Elaziz, M., T. Moriya, J. Akahira, T. Suzuki, and H. Sasano, StAR and progesterone producing enzymes (3beta-hydroxysteroid dehydrogenase and cholesterol side-chain cleavage cytochromes P450) in human epithelial ovarian carcinoma: immunohistochemical and real-time PCR studies. Cancer Sci, 2005. 96(4): p. 232-9.

35. Park, C.S., T.K. Kim, H.G. Kim, Y.J. Kim, M.H. Jeoung, W.R. Lee, et al., Therapeutic targeting of tetraspanin8 in epithelial ovarian cancer invasion and metastasis. Oncogene, 2016. 35(34): p. 4540-8.

36. Hanahan, D. and L.M. Coussens, Accessories to the crime: functions of cells recruited to the tumor microenvironment. Cancer Cell, $2012.21(3)$ : $\mathrm{p}$. 309-22.

37. Stolk, D., H.J. van der Vliet, T.D. de Gruijl, Y. van Kooyk, and M.A. Exley, Positive \& Negative Roles of Innate Effector Cells in Controlling Cancer Progression. Front Immunol, 2018. 9: p. 1990.

38. Taube, J.M., J. Galon, L.M. Sholl, S.J. Rodig, T.R. Cottrell, N.A. Giraldo, et al., Implications of the tumor immune microenvironment for staging and therapeutics. Mod Pathol, 2018. 31(2): p. 214-234.

39. Hui, L. and Y. Chen, Tumor microenvironment: Sanctuary of the devil. Cancer Lett, 2015. 368(1): p. 7-13.

40. Fridman, W.H., L. Zitvogel, C. Sautès-Fridman, and G. Kroemer, The immune contexture in cancer prognosis and treatment. Nat Rev Clin Oncol, 2017. 14(12): p. 717-734.

41. Mhaidly, R. and F. Mechta-Grigoriou, Fibroblast heterogeneity in tumor micro-environment: Role in immunosuppression and new therapies. Semin Immunol, 2020. 48: p. 101417.

42. Gao, Q., Z. Yang, S. Xu, X. Li, X. Yang, P. Jin, et al., Heterotypic CAF-tumor spheroids promote early peritoneal metastatis of ovarian cancer. J Exp Med, 2019. 216(3): p. 688-703

43. Nersesian, S., H. Glazebrook, J. Toulany, S.R. Grantham, and J.E. Boudreau, Naturally Killing the Silent Killer: NK Cell-Based Immunotherapy for Ovarian Cancer. Front Immunol, 2019. 10: p. 1782.

44. Malchiodi, Z.X. and L.M. Weiner, Understanding and Targeting Natural Killer Cell-Cancer-Associated Fibroblast Interactions in Pancreatic Ductal Adenocarcinoma. Cancers (Basel), 2021. 13(3).

\section{Figures}
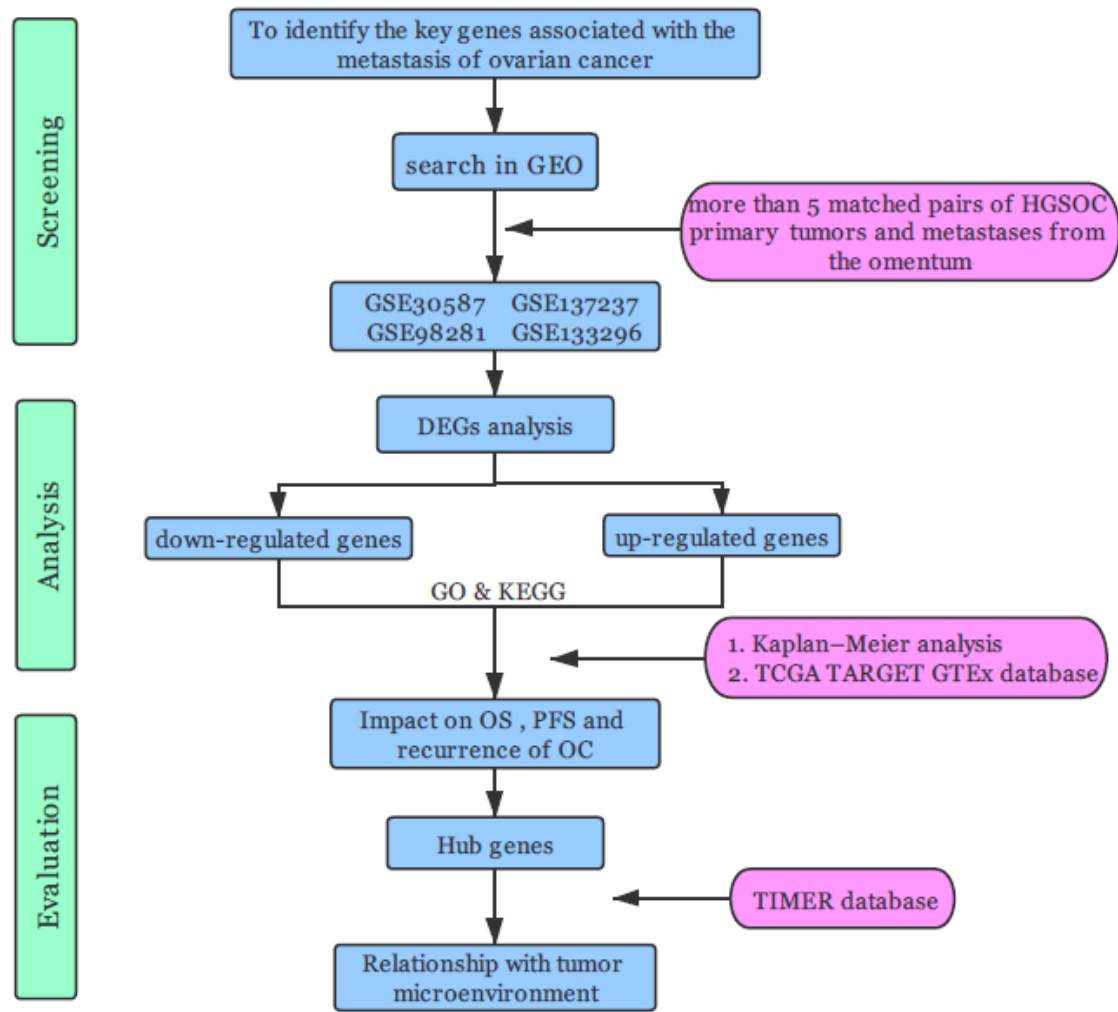

Figure 1

The work flow diagram of whole study design and analysis 


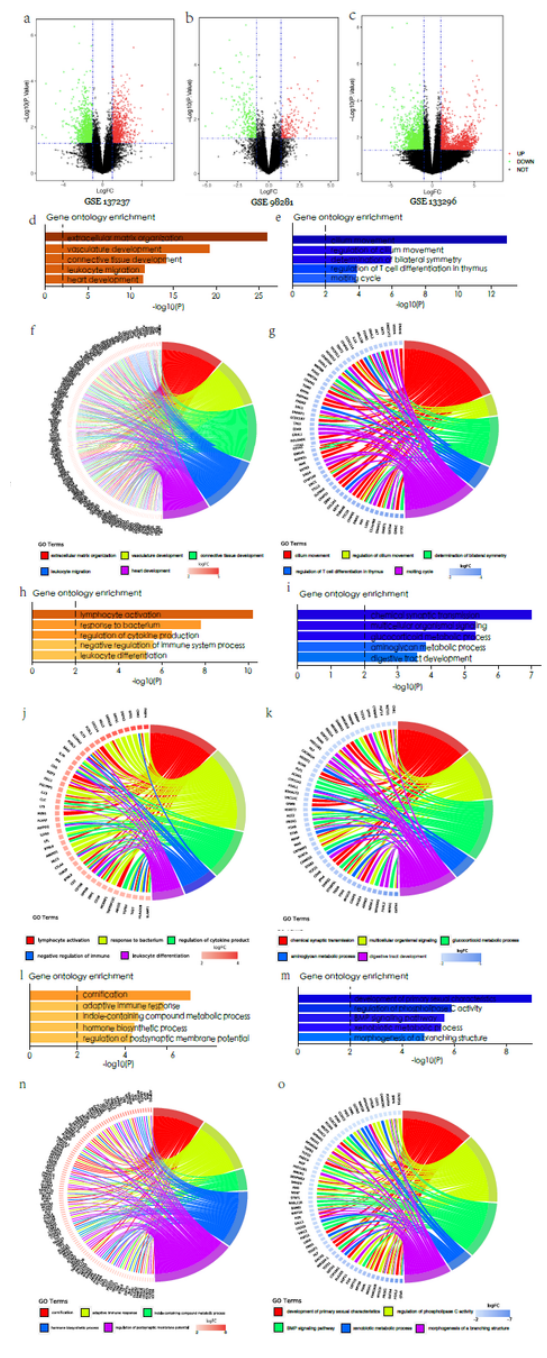

\section{Figure 2}

GO enrichment analysis of DEGs about every GEO data. (a) Volcano plot showing the DEGs between PT samples $(n=11)$ and MT samples ( $n=11)$ in GSE 137237 data. (b) Volcano plot showing the DEGs between PT samples $(n=10)$ and MT samples $(n=10)$ in GSE98281 data. (c) Volcano pl ot showing the DEGs between PT samples $(n=10)$ and MT samples $(n=10)$ in GSE133296 data. (d-g) The top 5 GO terms and distribution of up-regulated and down-regulated genes of MT group compared with PT group in GSE137237 data. (h-k) The top 5 GO terms and distribution of up-regulated and down-regulated genes of MT group compared with PT group in GES98281 data. (I-o) The top 5 GO terms and distribution of up-regulated and down-regulated genes of MT group compared with PT group in GSE133296 data. 
a

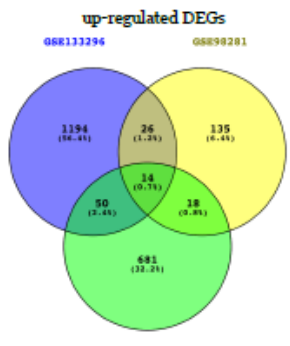

C

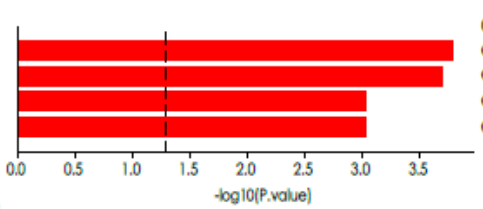

d

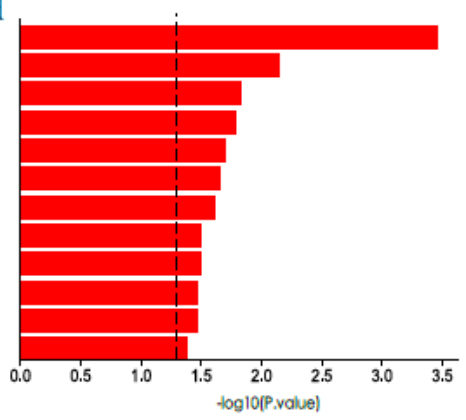

b

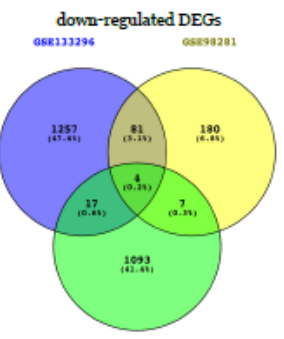

aระ13า23า

Gene ontology enriohment

GO:0030178:negative regulation of Wnt signaling pathway

GO:0045444,fat cell differentiation

GO:0030198:extracellular matrix organization

GO:0032102:negative regulation of response to external stimulus

hs003320:PPAR signaling pattway

hisa00100-Steroid biosynthesis

hsa04975:Fat digestion and absorption

hsa04930:Type II diabetes mellitus

hs 004923:Regulation of lipolysis in adipocytes

hsa00561:Glycerolipid metabolism

hsa04920:Adipocytokine signaling pattway

hsa04211:Longevity regulating pathway

is 004974: Protein digestion and absorption

hsa04640:Hematopoletic cell lineage

hsa04972:Pancreatic secretion

hsa04152:AMPK signaling pathway

Figure 3

Identification of candidate genes between primary ovarian cancer and matched metastatic omentum tumor. (a, b) Venn diagram of overlapping 14 upregulated genes and 4 down-regulated genes based on the three GEO datasets. (c, d) GO analysis and KEGG analysis of 14 up-regulated genes. 

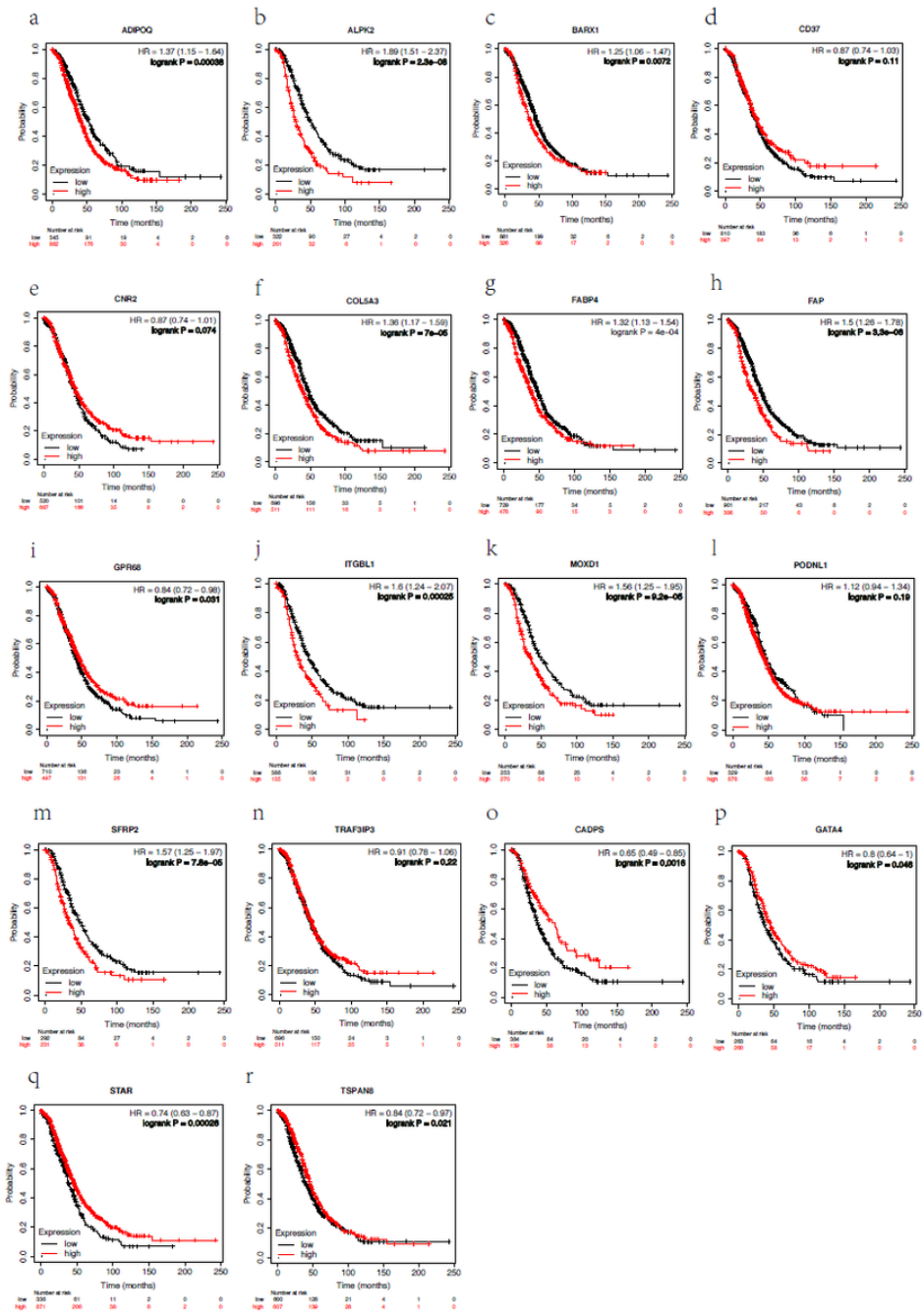

\section{Figure 4}

Overall survival (OS) analysis curves of hub genes. (a-n) The overall survival analysis of up-regulated genes (ADIPOQ, ALPK2, BARX1, CD37, CNR2, COL5A3, FABP4, FAP, GPR68 ITGBL1, MOXD1, PODNL1, SFRP2 and TRAF3IP3) in metastatic tumors. (o-r) The overall survival analysis of down-regulated genes (CADPS, GATA4, STAR and TSPAN8) in metastatic tumors. 

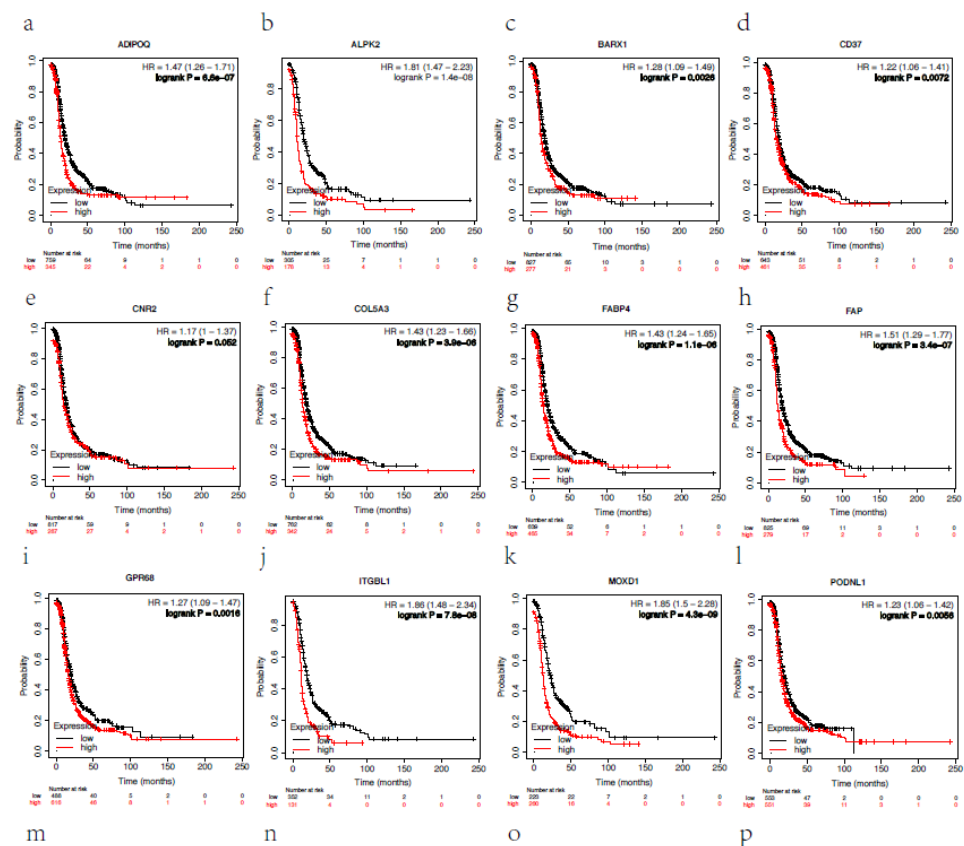

m
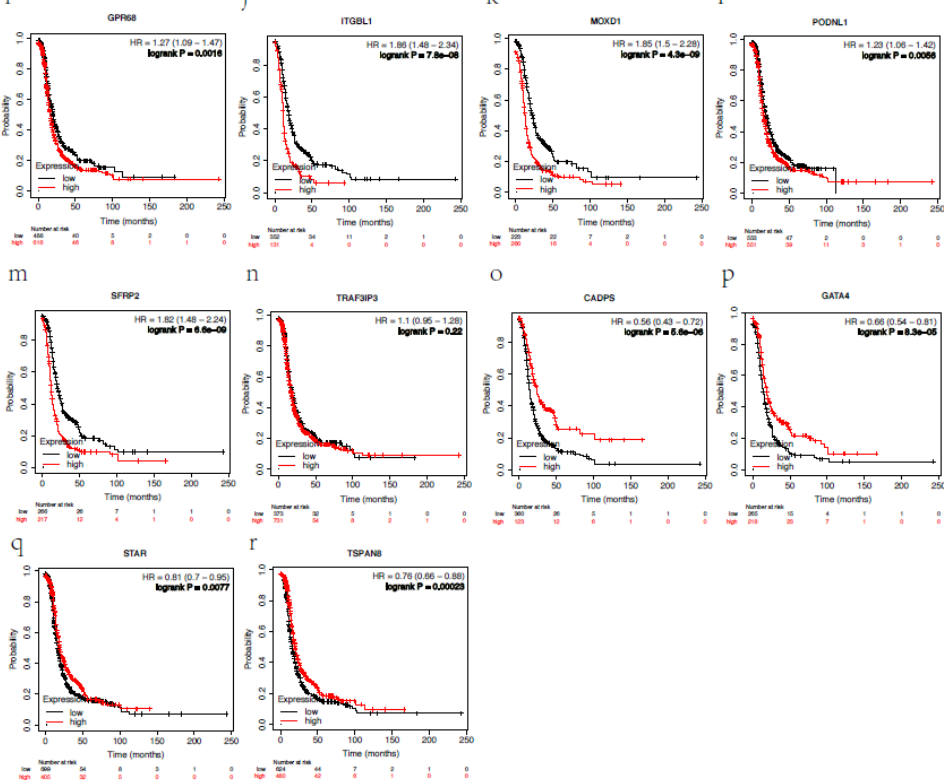

Figure 5

progression-free survival(PFS) analysis curves of hub genes. (a-n) The progression-free survival analysis of up-regulated genes in metastatic tumors. (o-r) The progression-free survival analysis of down-regulated genes in metastatic tumors. 

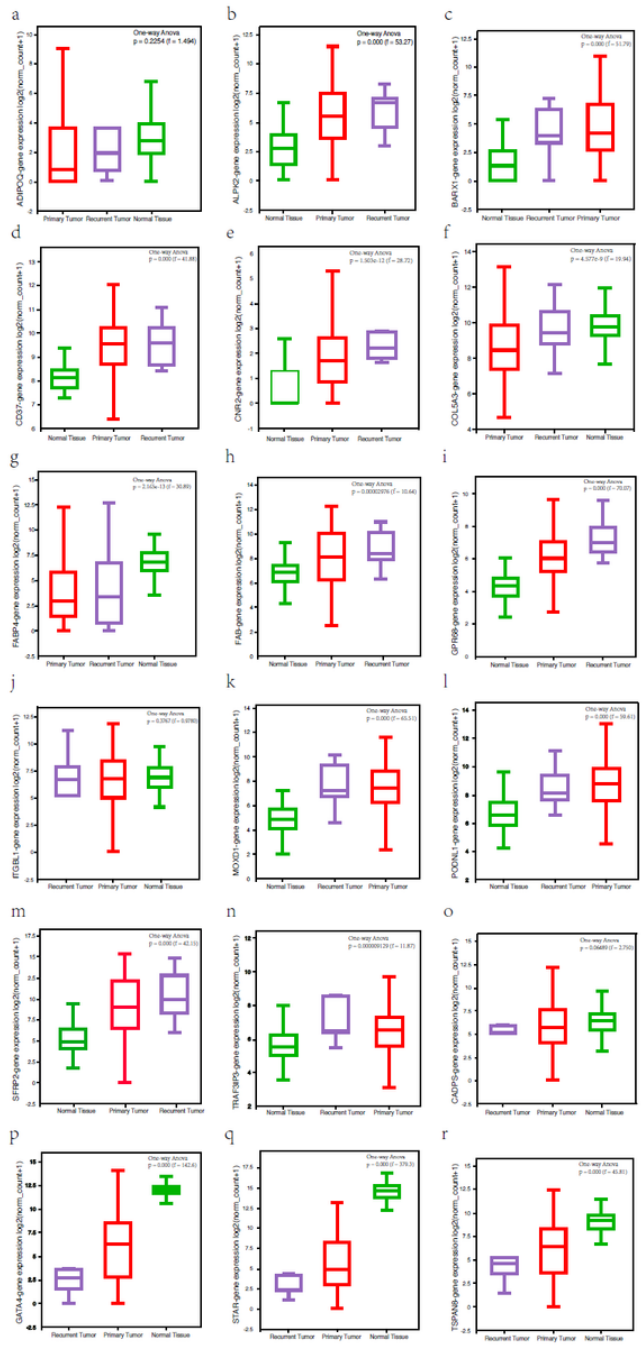

\section{Figure 6}

Evaluation of hub genes expression among normal ovary, primary tumors and recurrent tumors. (a-n) The different expression of up-regulated genes. (o-r) The different expression of down-regulated genes. 


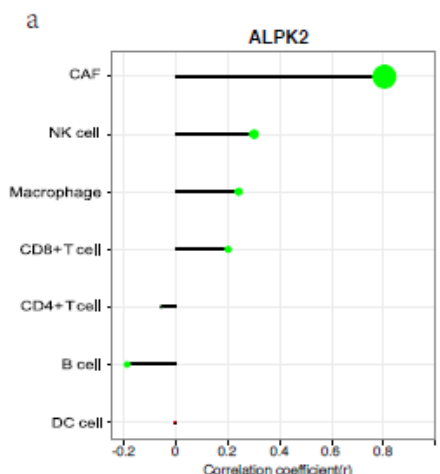

d

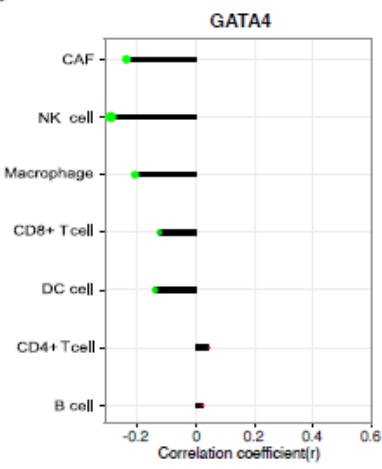

b

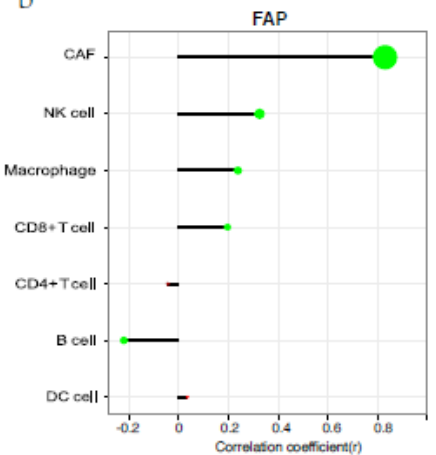

e

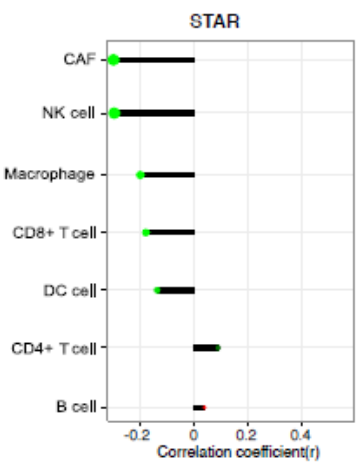

c

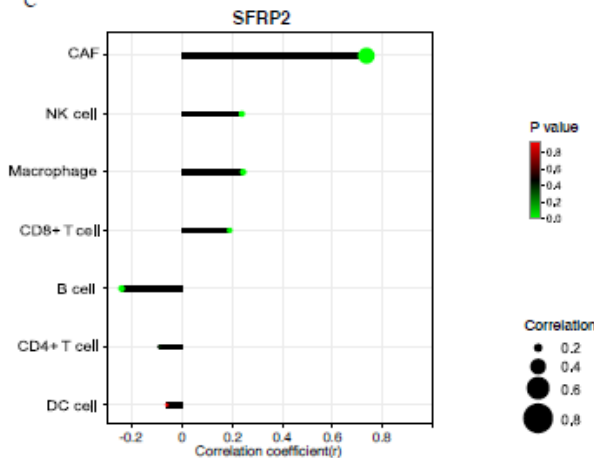

f

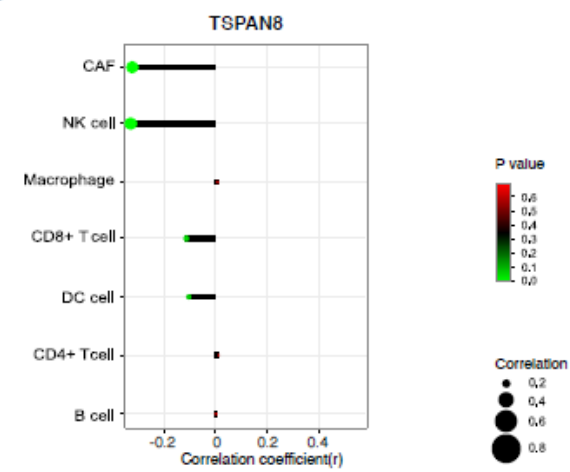

Figure 7

Correlation analysis between the expression of 6 hub genes and tumor microenvironmental cells infiltration level. (a)ALPK2, (b)FAP, (c)SFRP2, (d)GATA4, (e)STAR, (f)TSPAN8.

\section{Supplementary Files}

This is a list of supplementary files associated with this preprint. Click to download.

- AdditionalfigS1Standardizationofgeneexpression.pdf

- Additionalfigs2.pdf

- AdditionalfigS3.pdf

- Additionalfigureandtablelegends.docx

- Additionaltables1.xlsx

- Additionaltables2.xlsx

- AdditionaltableS3.xlsx

- AdditionaltableS4.xlsx

- AdditionaltableS5.xlsx 